\title{
El antiguo kuracazgo de Tacna: llactas, mandones y encomenderos (siglo XVI)
}

\author{
The ancient kuracazgo of Tacna: llactas, mandones y encomenderos, in the 16th \\ century
}

${ }^{1}$ Adán Umire Alvarez

ORCID 0000-0002-1707-4896

\section{RESUMEN}

En el siglo XVI, durante el reparto de encomiendas realizado por el Marqués Francisco Pizarro, estas fueron entregadas con sus respectivos elementos productivos: la extensión de tierras y la cantidad de gente como mano de obra disponible. Esta riqueza en el kuracazgo de Tacna, de acuerdo a los antecedentes de la investigación, tuvo una estructura de gobierno en base a dos mitades: Hanansaya con su llacta Pachía y su mandón Ccata, Cata o Cato, concedida a Hernando de Torres; y Urinsaya con la llacta Tacana y su mandón Estaca o Istaca concedida a Pedro Pizarro. De una nueva aproximación a las fuentes originales, se demuestran dos aspectos importantes: que la concesión de las sayas a los encomenderos mencionados tiene un orden equivocado, y que el kuracazgo de Tacna nunca ha presentado una estructura unitaria dual andina, esta fue el resultado de una forma de gobierno impuesta por la autoridad española. Se argumenta la ausencia de la oposición dual andina hanan/urin en el valle de Tacna, por la falta de elementos Inka tangibles (estructuras domésticas, militares, religiosas). Se concluye sugiriendo que debieron existir otros sistemas de organización andina en el valle de Tacna, previo a la llegada de Inkas y españoles.

Palabras clave: Encomienda, estructura dual andina, kuracazgo de Tacna.

\section{ABSTRACT}

In the 16th century, during the distribution of encomiendas carried out by the Marquis Francisco Pizarro, he delivered each of them with its productive elements made up of the extension of land and the number of people as available labor. This wealth in the kuracazgo of Tacna, according to the backgrounds of the research, had a government structure based on two halves: Hanansaya with its llacta Pachía and its mandón Ccata, Cata or Cato, granted to Hernando de Torres; and Urinsaya with the llacta Tacana and its mandón Estaca or Istaca granted to Pedro Pizarro. From a new approach to the original sources, two important aspects are demonstrated: that the granting of the sayas to the mentioned encomenderos has a wrong order, and that the kuracazgo of Tacna has never presented a dual Andean unitary structure, this was the result of a form of government imposed by the Spanish authority. The absence of the dual Andean hanan/urin opposition in the Tacna valley is argued, due to the lack of tangible Inka elements (domestic, military, religious structures). It is concluded by suggesting that there must have been other Andean organization systems in the Tacna valley, prior to the arrival of Inkas and Spaniards.

Key words: Encomienda, Andean dual structure, kuracazgo of Tacna.

${ }^{1}$ Universidad Católica San Pablo, Centro de Estudios Peruanos. Arequipa, Perú. E-mail: adan.umire.alvarez@ucsp.edu.pe 


\section{INTRODUCCIÓN}

Después de la invasión, durante el reparto de tierras en encomiendas por parte de la Corona española a todos los participantes en la conquista de las tierras del sol, se dispuso que cada región y encomienda debería contar con una cantidad adecuada de tierras y sus respectivas poblaciones indígenas habitantes. Que en definitiva "tierra (medio productivo) y gente (mano de obra)" resuman la riqueza de dicha encomienda. Así, la organización política de estas comunidades, de acuerdo a la lectura de los antecedentes para esta investigación, se resume en $a$ ) una estructura de gobierno unitaria en base a dos mitades, traducida en $b$ ) una dualidad en sayas, con una llacta como cabeza de cada una de las mitades existentes en el valle de Tacna.

Del análisis sobre organización andina prehispánica del kuracazgo de Tacna, llevada a cabo desde la perspectiva etnohistórica (Cavagnaro, 1986; Cúneo, 1977) e intentos propugnados desde una visión arqueológica (Gordillo, 2014), resumimos la primera posición donde el documento de previsión otorgada por el Marqués Francisco Pizarro a los encomenderos Pedro Pizarro y Hernando de Torres refleja una dualidad tácita: los Hanansaya con su llacta Pachía y su mandón Ccata, Cata o Cato; y la llacta Tacana con su mandón Estaca o Istaca de la mitad Hurinsaya. De la información resumida, se desprende lo siguiente: que para ubicar en la actualidad la correspondencia de cada una de las dos mitades mencionadas, la parte media superior del valle de Caplina, o Hanansaya, le fue otorgada a Hernando de Torres y la parte baja, o Hurinsaya, desde Tacna hasta la costa del mar a Pedro Pizarro.

No obstante, de la lectura de fuentes adicionales (Barriga, 1939; Barriga, 1955), se desprende que la encomienda de Hernando de Torres se extendía hasta Boca del río Zama incluyendo a pueblos de pescadores; por lo que la llacta Pachía, ubicada al este de Tacna, no estuvo encomendada a Hernando de Torres ¿Será posible que las ubicaciones de las sayas tuvieran una lectura de posición geográfica equivocada, que en este caso habrían sido interpretadas tácitamente, sin mucho rigor espacial?

En cuanto al tema de la organización andina en Tacna, con las dos sayas y sus respectivas llactas, parece conducir a la conclusión de que el valle de Tacna en 1538-1540 estuvo gobernado por dos caciques principales bajo una "estructura unitaria dual". Si bien, la ausencia de mayores datos para sustentar esta estructura es evidente, en este artículo se argumenta que la "estructura unitaria dual" sugerida para el kuracazgo de Tacna, en investigaciones anteriores (Cavagnaro, 1986; Cúneo 1977), fue una solución impuesta por los españoles durante el repartimiento de tierras a los conquistadores, no constituyendo una división ancestral andina, como más adelante detallaremos.

\section{CONCEPTO DE DUALIDAD}

En la introducción de este tema, se debe tomar en cuenta la división decimal de la población cuya función socio-económica fue introducida por el Estado Inka para trabajos agropecuarios, obras estatales, servicio militar o centros de producción, frente a otra división más bien territorial y de oposición dual (hanan/urin) que se yuxtapone a la división decimal (Arellano, 1989).

Esta división territorial de oposición dual hanan/urin, en la que la primera significaba "arriba" y la siguiente "abajo", también ha podido tener un sentido valorativo antagónico de superior/inferior, como sospecha Arellano para los Andes centrales y se hace extensivo hacia nuestra zona de estudio (Arellano, 1989). Al parecer, es en este último sentido que Cúneo haya tomado las informaciones históricas para presentar sus apreciaciones:

"Este Catari Apassa aparece como Ccata (síncopa de Ccatari) en la cédula, fechada en el Cuzco a 2 de noviembre de 1538, por la cual el dicho marqués Pizarro encomienda 
600 indios del valle de Tacna en Pedro Pizarro..."(Cúneo, 1977, p. 325).

"Se echa de ver que, desaparecida la hegemonía que Chucuito venía ejerciendo desde época inmemorial...el de Tacna, habiase fragmentado en dos cacicazgos menores, sobre la pauta de sus ancestrales divisiones de casta" (Cúneo, 1977, p. 326, el subrayado es nuestro).

Este esquema de división dual de oposición territorial parece que no fue la única en el mundo andino; como menciona Arellano, hubo otras divisiones para la sierra central del Perú como curac/sullca, hatun/huchuy; hatun/ura, allauka/ichoq (Arellano, 1989) que se podrían traducir como términos antagónicos y a la misma vez complementarios: mayor/menor, grande/pequeño, arriba/abajo, etc. De la misma forma, por la presencia de expresiones culturales arqueológicas del Período Intermedio Tardío, como San Miguel y Gentilar, es posible que en el valle de Caplina también funcionaron algunas otras divisiones previas y ajenas a la dualidad conocida para la época Inka.

Si el Estado Inka hubiese introducido en el valle de Tacna la división dual de oposición hanan/urin, esto debiera reflejarse en una presencia directa de vestigios arqueológicos para entender exactamente su significado (Arellano, 1989). Salvo algunos elementos funerarios Inka en Peañas, Cristo Rey y pocos fragmentos cerámicos superficiales en otros sitios arqueológicos del mismo valle de Tacna, no hay mayores indicadores estructurales que permitan inferir la presencia de entidades estatales Inka de control territorial. En cambio, en ambientes costeros como Cerro Los Hornos (Vela, 2004), Sama La Antigua (Trimborn, 1975), Tacahuay (Chacaltana, 2015), y ambientes más alejados como Putina, Palca y Moqui (Gordillo, 2014; Gordillo y Bolaños, 2019; Vaughan, 2013), donde existen evidencias con estructuras posiblemente de carácter administrativo, podrían haber funcionado perfectamente la división de oposición introducida e impuesta por el Estado Inka sobre otras divisiones locales mucho más antiguas.

\section{ELREPARTIMIENTO DE TACNA}

De las informaciones revisadas (Barriga, 1939; Barriga, 1955) se desprende que las encomiendas adscritas a Pedro Pizarro y Hernando de Torres ocupaban un ambiente geográfico con poblaciones dispersas a lo largo del valle, que pueda entendérsela como valle alto y bajo además de la extensa y desierta playa costera; que este espacio limita por el norte con la entonces bien poblada Boca del Río (Sama) y por el sur hasta las inmediaciones de Arica. La extensión de la faja costera, incluida en esta encomienda, quizás se entienda por la ausencia de verdaderos asentamientos poblacionales, desde el abanico fluvial seco de la parte baja de Tacna hasta Boca del Río (Sama) (23 km aproximadamente) y desde el mismo abanico fluvial hasta la desembocadura de Lluta (26 $\mathrm{km})$.

Para explicar esta situación, en el valle de Tacna sucedieron algunos hechos de interés durante la entrega de encomiendas. Durante el censo, antes de 1540, parece que hubo muchos pueblos de indios dispersos a lo largo y ancho del valle de Tacna (Tabla 1), con una cantidad considerable de habitantes y con la promesa de ser entregada completamente a Pedro Pizarro. Al momento de entregarla en 1540, las promesas iniciales no fueron cumplidas, y el valle de Tacana (dividida en forma arbitraria) fue encomendada a dos españoles participantes de la conquista: Pedro Pizarro y Hernando de Torres. La razón expuesta para justificar este hecho es la previsión de gente para el poblamiento de "Villa Hermosa" [iel valle de Camaná, o Arequipa?] por ser puerto. A Pedro Pizarro se le otorgó la mitad del valle de Tacna con 800 indios y a Hernando de Torres la otra mitad con 600 (Barriga, 1939). Por circunstancias que no vamos a revisar aquí, Pedro Pizarro, después de tres años de mantenerla (1543), cede su parte a Hernando de Torres; volviéndola después a recuperar en 1559 (Barriga, 1940). 
El antiguo kuracazgo de Tacna: llactas, mandones y encomenderos (siglo XVI)

Julio-diciembre 2020

Tabla 1

El repartimiento del valle de Tacna

VALLE DE TACNA

\begin{tabular}{|c|c|c|c|c|c|c|c|}
\hline \multirow[b]{2}{*}{ Cacique } & \multirow{2}{*}{$\begin{array}{l}\text { Pueblo o lugar } \\
\text { mencionado en } \\
\text { el documento }\end{array}$} & \multirow{2}{*}{$\begin{array}{c}\text { Localización } \\
\text { señalada en } \\
\text { el } \\
\text { documento }\end{array}$} & \multirow{2}{*}{$\begin{array}{l}\text { Lugar } \\
\text { geográfico } \\
\text { actual }\end{array}$} & \multirow[t]{2}{*}{ Población } & Jerarquía & \multicolumn{2}{|c|}{ Mitimaes } \\
\hline & & & & & Mandón Principal & Sí & No \\
\hline $\begin{array}{l}\text { Istaca o } \\
\text { Astaca }\end{array}$ & Pueblo de Tacana & $\begin{array}{l}\text { Pueblo de } \\
\text { Tacana? }\end{array}$ & $\begin{array}{l}\text { Valle de } \\
\text { Tacna }\end{array}$ & $\begin{array}{l}800, \\
\text { otorgado a } \\
\text { Pedro } \\
\text { Pizarro en } \\
1542\end{array}$ & Mandón & No & \\
\hline $\begin{array}{l}\text { Quelopano, } \\
\text { Quilopana }\end{array}$ & $\begin{array}{l}\text { Pueblo de } \\
\text { Tacana? }\end{array}$ & $\begin{array}{l}\text { Pueblo de } \\
\text { Tacana? }\end{array}$ & $\begin{array}{l}\text { Valle de } \\
\text { Tacna }\end{array}$ & & Principal & No & \\
\hline Omechipa & & $\begin{array}{l}\text { Valle de } \\
\text { Tacana? }\end{array}$ & $\begin{array}{l}\text { Valle de } \\
\text { Tacna }\end{array}$ & & Principal & No & \\
\hline $\begin{array}{l}\text { Llanchipa, } \\
\text { Lanchita }\end{array}$ & & $\begin{array}{l}\text { Valle de } \\
\text { Tacana? }\end{array}$ & $\begin{array}{l}\text { Valle de } \\
\text { Tacna }\end{array}$ & 600 & Principal & No & \\
\hline Condori & & $\begin{array}{l}\text { Valle de } \\
\text { Tacana? }\end{array}$ & $\begin{array}{l}\text { Valle de } \\
\text { Tacna }\end{array}$ & & Principal & No & \\
\hline $\begin{array}{l}\text { Concharque, } \\
\text { Concharique }\end{array}$ & & $\begin{array}{l}\text { Valle de } \\
\text { Tacana? }\end{array}$ & $\begin{array}{l}\text { Valle de } \\
\text { Tacna }\end{array}$ & & $\begin{array}{l}\text { Principal, en } 1543 \text { se } \\
\text { convierte en } \\
\text { mandón? }\end{array}$ & No & \\
\hline Quilla, Quiela & & $\begin{array}{l}\text { Valle de } \\
\text { Tacana? }\end{array}$ & $\begin{array}{l}\text { Valle de } \\
\text { Tacna }\end{array}$ & & Principal & No & \\
\hline Cata o Cato & & $\begin{array}{l}\text { Valle de } \\
\text { Tacana? }\end{array}$ & $\begin{array}{l}\text { Valle de } \\
\text { Tacna }\end{array}$ & $\begin{array}{l}600 \\
\text { otorgado a } \\
\text { Hernando } \\
\text { de Torres } \\
\text { en } 1542 \\
\end{array}$ & Segundo Mandón? & No & \\
\hline Talassi & Valle de Tacna & $\begin{array}{l}\text { Mezclados en } \\
\text { Tacana }\end{array}$ & $\begin{array}{l}\text { Valle de } \\
\text { Tacna }\end{array}$ & 40 & Principal & No & \\
\hline Talassi? & $\begin{array}{l}\text { Pescadores en } \\
\text { Boca del rio } \\
\text { Zama e } \\
\text { Capanique }\end{array}$ & $\begin{array}{l}\text { Boca de Río } \\
\text { Sama }\end{array}$ & $\begin{array}{l}\text { Boca de } \\
\text { Río }\end{array}$ & & Principal & No & \\
\hline Soco & Pueblo Chichi & $\begin{array}{l}\text { Valle de } \\
\text { Tacana? }\end{array}$ & $\begin{array}{l}\text { Valle de } \\
\text { Tacna }\end{array}$ & 27 & Principal & No & \\
\hline Maqui & Anaquina & $\begin{array}{l}\text { Valle de } \\
\text { Tacana? }\end{array}$ & $\begin{array}{l}\text { Valle de } \\
\text { Tacna }\end{array}$ & 6 & Principal & No & \\
\hline Sucutila & Arica & $\begin{array}{l}\text { Arica? o } \\
\text { costa de } \\
\text { Tacana? }\end{array}$ & Arica? & 8 & Principal & No & \\
\hline Chica & & $\begin{array}{l}\text { Valle de } \\
\text { Tacana? }\end{array}$ & $\begin{array}{l}\text { Valle de } \\
\text { Tacna }\end{array}$ & & Principal & No & \\
\hline Caena & & $\begin{array}{l}\text { Valle de } \\
\text { Tacana? }\end{array}$ & $\begin{array}{l}\text { Valle de } \\
\text { Tacna }\end{array}$ & & Principal & No & \\
\hline Pay & & Valle de & Valle de & & Principal & No & \\
\hline
\end{tabular}




\begin{tabular}{|c|c|c|c|c|c|}
\hline & & Tacana? & Tacna & & \\
\hline Cabano & & $\begin{array}{l}\text { Valle de } \\
\text { Tacana? }\end{array}$ & $\begin{array}{l}\text { Valle de } \\
\text { Tacna }\end{array}$ & $\begin{array}{l}\text { Principal, pero } \\
\text { manda en lugar de } \\
\text { Cato }\end{array}$ & No \\
\hline $\begin{array}{l}\text { Talasi, es el } \\
\text { mismo } \\
\text { Talassi? }\end{array}$ & $\begin{array}{l}\text { Capanique es } \\
\text { pueblo en Tacna }\end{array}$ & $\begin{array}{l}\text { Valle de } \\
\text { Tacana? }\end{array}$ & $\begin{array}{l}\text { Valle de } \\
\text { Tacna }\end{array}$ & $\begin{array}{l}\text { Pero se ha tomado } \\
\text { algunos indios }\end{array}$ & No \\
\hline Coaquiera & & $\begin{array}{l}\text { Valle de } \\
\text { Tacana? }\end{array}$ & $\begin{array}{l}\text { Valle de } \\
\text { Tacna }\end{array}$ & $\begin{array}{l}\text { Principal, hermano } \\
\text { de Talassi }\end{array}$ & No \\
\hline Tucuba & & $\begin{array}{l}\text { Valle de } \\
\text { Tacana? }\end{array}$ & $\begin{array}{l}\text { Valle de } \\
\text { Tacna }\end{array}$ & Principal & $\begin{array}{l}\text { Sí, son de } \\
\text { Tarapacá }\end{array}$ \\
\hline
\end{tabular}

Fuente: Barriga, 1939 (Provisión del marqués Francisco Pizarro concediendo la encomienda de Tacna, Curane y sus parcialidades, al conquistador Pedro Pizarro, y parte a Hernando de Torres).

En resumen, las encomiendas de Tacna en 1540 quedarían de la siguiente forma:

a) Encomienda de Pedro Pizarro, conformada por 800 indios NO MITIMAES y su mandón Istaca, además de:

- Principal Quelopano, en el valle de Tacana.

- Principal Llanchipa, en el valle de Tacana.

- Principal Condori, en el valle de Tacana.

- Principal Concharque, en el pueblo de Tacana.

- Principal Quilla, en el pueblo de Tacana.

Se deduce que los principales Concharque y Quilla se ubican en el pueblo de Tacna, esta información sugiere que ambos principales vivían dentro de los límites del pueblo. En cambio, los restantes principales vivían en otros lugares o poblados, aunque en el mismo valle de Tacna.

b) Encomienda de Hernando de Torres, con el segundo mandón Cata o Cato y 600 indios; además de los MITIMAES compuesto por el mandón Cariapassa (¿en Azapa o Arica?) y conformada por:

- Principal Tucuba, con indios de Tarapacá, ¿en el valle de Tacana?

- Principal Talassi, en valle de Tacana, servía a Capanique (¿pueblo?).

- Principal Talassi y pescadores de la boca del río Zama, Boca de Río Sama.

- Principal Soco del pueblo de Chichi, ¿en Tacana? ${ }^{2}$

- Principal Maqui del pueblo de Anaquina, ¿en Tacana?

- Principal Sucutila del pueblo de Arica, ¿en Tacana? No menciona que es pueblo de pescadores o costa de la mar

- Principal Chica, ¿en el valle de Tacana?

- Principal Caena, ¿en el valle de Tacana?

- Principal Pay, ¿en el valle de Tacana?

- Principal Cabano, ¿en el valle de Tacana?

- Principal Coaquiera, ¿en el valle de Tacana?

De esta relación se prevé tres grupos de comunidades cuyos lugares de residencia estuvieron caracterizados por diferentes ambientes geográficos: a) coincidente con el pueblo actual de Tacna, b) con el valle de Tacna, y c) con la línea costera y Boca del Río (Sama). En ese sentido, para los principales Soco, Maqui, quizá Sucutila, se menciona como posible lugar de residencia al Pueblo de Tacna; Tucuba, Chica, Caena, Pay, Cabano, Coaquiera y Talassi en el valle de Tacna (este último específicamente en Capanique). Nuevamente, Talassi en Boca del Río (Sama); de lo que se deduce que Capanique pudo ubicarse al

\footnotetext{
${ }^{2}$ Aunque Hidalgo $(1996 ; 2004)$ transcribe el nombre del principal como 'Seel Soco', se cree que aquí hubo un error del copista/escribano de la época de transcripción moderna, toda vez que los nombres de todos los mandones y principales están conformados por una palabra. En este caso si nuestra observación es correcta. En lugar de ser "Seel Soco" debería ser "deel" o sea "de él" que denota propiedad, como en los otros casos donde se adscribe pertenencia del principal a su pueblo.
} 
occidente del pueblo de Tacna, es decir, en la parte baja del valle, quizá próximo a Boca del Río Sama. Del mismo modo, las actividades de Talassi podrían ser ambas: pescador y agricultor.

La cantidad de pueblos y sus respectivos habitantes en Tacna casi cuadruplicaron en número que los del valle de Azapa y superaron ampliamente al valle y costa de Ilo juntos, lo que la hace importante al momento de concesión en encomienda. Quizás este hecho hizo que el valle de Tacna fuera dividida y entregada a dos encomenderos: Pedro Pizarro y Hernando de Torres.

Cúneo-Vidal (1977), después de resumir el documento de la previsión en la que solo considera beneficiario a Pedro Pizarro, concluye que el mandón Ccata, o Cata o Cato y su pueblo pertenecen a los Hanansayas con su llacta Pachía (o establecimiento principal) y la llacta
Tacana con su mandón Estaca (Istaca de la encomienda de Pedro Pizarro) al de Urinsaya. En este resumen, la parte media superior $\mathrm{u}$ oriental del valle de Tacna le fue otorgada a Hernando de Torres y la parte baja hasta la costa del mar a Pedro Pizarro. Sin embargo, a la luz del análisis crítico y relectura de otras fuentes históricas, el resumen de Cúneo-Vidal no parece ser correcto y la ubicación de ambas mitades y sus encomenderos es más bien contraria.

Una de las encomiendas se extendía desde la mitad del valle de Tacna hasta la boca del río Sama. De los asentamientos que comprendían la encomienda de Hernando de Torres, el principal Talassi servía tanto en Capanique como en Boca del río Zama, junto con los pescadores, por lo que se deduce que la encomienda de Hernando de Torres pudo haberse extendido desde la mitad hacia la parte baja del valle de Tacna e incluía hasta lo que hoy día conocemos como el centro

\section{Figura 1}

Vista panorámica del valle de Caplina y la disposición de las encomiendas, en la que se incluye los lugares actuales de Tacna y Pachía.

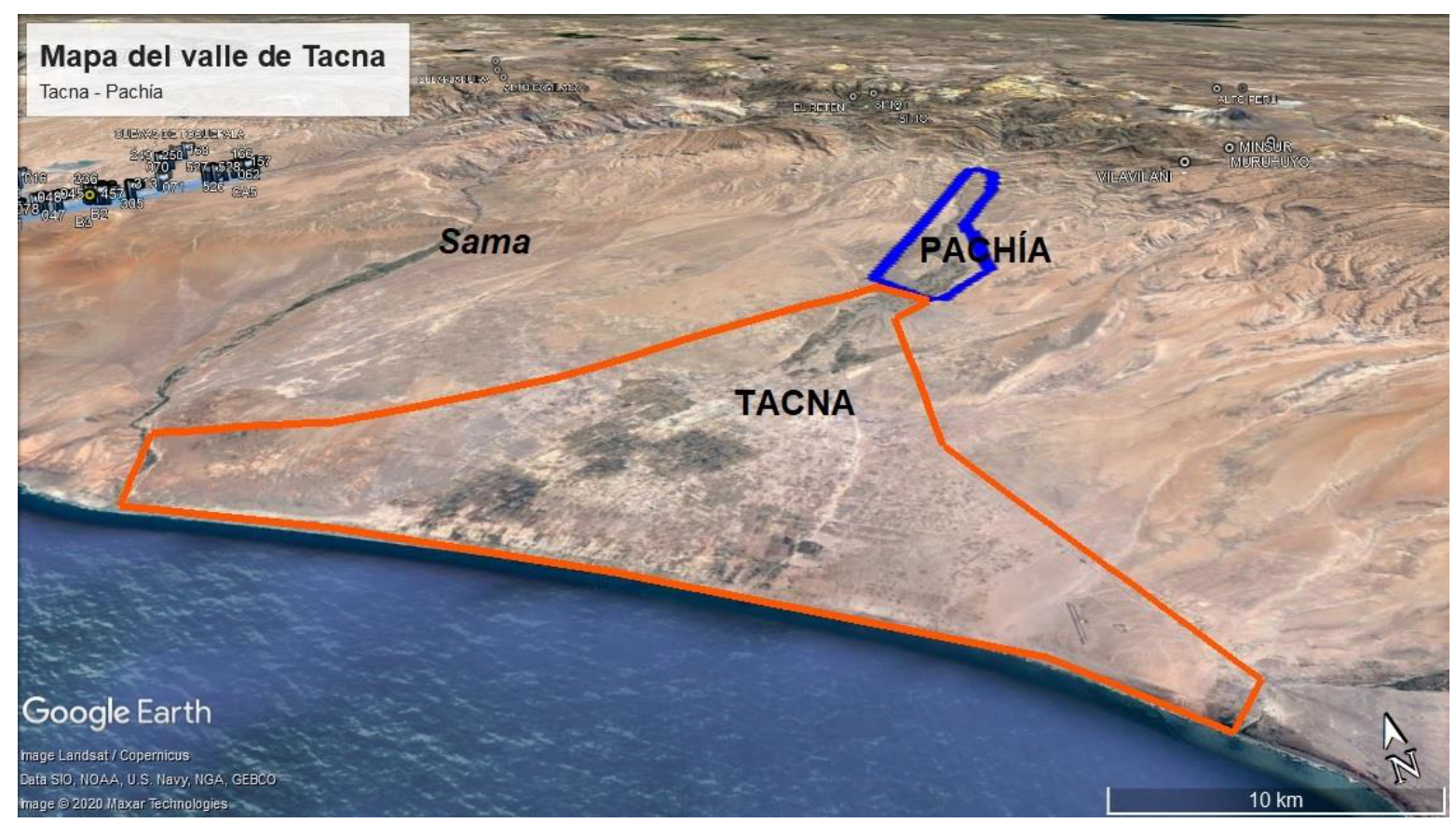

Nota. Se puede observar la posición geográfica de ambos lugares y su proximidad al mar y extensión hasta Boca del Río, en el caso de Tacna; a la misma vez, la posición de Pachía en la cabecera del valle, con abundancia de agua y terrenos agrícolas disponibles. 
poblado menor Boca del Río (Sama Las Yaras). En cambio, la encomienda de Pedro Pizarro incluía los pueblos actuales del sector oriental del valle de Tacna, como Pachía, Calana, y Calientes, quizás hasta Pallagua (Figura 1).

\section{ELPUEBLO DE CAPANIQUE}

Acerca de Capanique (también pudo denominarse Copanique), este es un ayllu "lindero" ubicado entre el límite de las parcialidades primitivas de Hanansaya y Hurinsaya (Cavagnaro, 1994). Hidalgo (2004) sugiere que pudo haber sido un cacique que posteriormente se convirtió en un ayllu con vínculos a una organización étnica mayor ubicada en el altiplano, convirtiéndola en un grupo mitma de acuerdo a la lectura de una cédula. Después de analizar la misma fuente revisada por Hidalgo, se advierte que Capanique es un pueblo cuyo principal fue Talassi, como así lo prueba el documento donde Pedro Pizarro toma nueva posesión de este pueblo en el valle de Tacna en 1559:

"...por quanto de mucho tiempo a esta parte el [Pedro Pizarro] tenia o poseía quieta y pacificamente la encomienda de los indios que al presente tiene e posee que es el repartimiento de Tacana con todos los caciques e principales a el subjetos con el pueblo nombrado Capanique que está en el dicho valle de Tacana..." (Barriga, 1940, $\mathrm{p}$. 285)

Lo que resalta de este hecho, es que Talassi ejerce autoridad tanto en los pescadores de la Boca del Río Sama como en el pueblo de Capanique, que suponemos es de agricultores, ubicada como límite entre Hanansaya y Urinsaya, es decir, entre el espacio que pueda corresponder en general a una posible división de pescadores y agricultores. La importancia de Capanique parece radicar en ser el pueblo principal de la encomienda de Pedro Pizarro. Más allá de esta breve explicación, con la información existente del pueblo Capanique no es posible proyectarse a otras hipótesis, salvo que desde el aspecto lingüístico el sufijo ique o ica pueda estar muy vinculado al habla de los pescadores, que en este caso podría sugerir algunos interrogantes sobre el principal Talassi y su rol de mandón bajo el cual se desenvuelven pescadores y algunos agricultores ${ }^{3}$.

Sobre el origen del ayllu Capanique en el valle de Tacna, es posible forzar una hipótesis. Hidalgo hace alusión a un documento tardío de 1594, donde el visitador de tierras Alonso García Ramón ordenó que la parcialidad ubicada en la quebrada de Estique deba bajar a Tacna “...por no poder acudir a la doctrina $y$ demás ministerios que eran obligados por estar [...] catorce leguas del pueblo de Tacna donde tienen su reducción" (Hidalgo, 2004, p. 548). En los cerros colindantes a Estique Pueblo (que viene a ser la zona poblada de mayor antigüedad y tradición) se encuentran varios asentamientos arqueológicos del período Intermedio Tardío, de los que sobresalen Pukara Q'eukela, Musata A y $B$, Picasa $A$ y $B$ cuya filiación cerámica está dada por el estilo denominado Sitajara (Gordillo, 1989, Estuquiña para Moquegua o Charcollo para la sierra de Arica); con algunos pocos fragmentos dispersos de cerámica San Miguel y Gentilar, especialmente en Picasa B. Quizás esta población originaria realizó el pago de 900 pesos y el compromiso de asistir a Tarata, por su cercanía y altitud, para recibir los oficios religiosos con la finalidad de que se les permita seguir residiendo en Estique. Es posible que, los descendientes de este grupo de agricultores y pastores prehispánicos fueran reducidos en Tacna o, en todo caso, trabajaban los campos de cultivo en el espacio de la reducción dentro del valle de Tacna, confirmándose la presencia de

${ }^{3}$ Hidalgo (2004) insiste en ver mitades en los pueblos de Tacana, sobre un error de lectura que hace del documento donde Pedro Pizarro es confirmado como posesionario del pueblo de Capanique en Tacna (Barriga, 1940). Escribe Tasali en vez de Talasi [esta vez con una "ese"], creando una dualidad ficticia, además de reemplazar a Tasali como cacique de Capanique. Además, perfila una unidad cacical formada por las dos mitades encabezadas por Istaca y Cata, que vienen a dividirse en cacicazgos independientes, y que supuestamente a su vez cada uno por su lado podría estructurarse internamente (Hidalgo, 2004). 
un ayllu aparentemente serrano en el poblamiento de la reducción colonial.

Por otro lado, llama la atención que dentro de la encomienda de De Torres se mencione a un principal Sucutila con su pueblo Arica. Pensamos que no se refiere al pueblo de Ariaca en la costa de la mar (actual puerto de Arica, en la parte baja de Azapa); parece que en la faja costera habitable paralelo al mar, como se ha venido mencionando, por razones que aún no sabemos (geográficas, topográficas, sociales, etc.) se nombran como Arica, Ariaca o Parica y que no corresponden a un solo sitio, sino a varios con similares características. Es posible que el topónimo de Arica designe algunas zonas específicas con sectores rocosos puntiagudos, con acumulaciones de guano de ave marina. Se debe tomar en cuenta que el significado del sufijo "ica" que eventualmente se repite, como en Ica, Arica, Ique-ique, podría corresponder a un marcador geográfico que señale una agrupación, reunión o conjunto de algo, relacionado al ambiente marítimo.

Tan al igual que los mitimaes de Tarapacá, mencionados para el pueblo Ariaca de pescadores del valle de Azapa, en Tacna también hubo un pueblo de mitimaes. El mitimae mandón Cariapassa ya tenía tres pueblos bajo su mando en el valle de Azapa, incluso uno era de pescadores en Arica y correspondía a indios de Tarapacá. Parece que de la misma suerte, en el valle de Tacna, Cariapassa es mandón de un pueblo cuyo principal es Tucuba, también con indios de Tarapacá (¿Quizás pescadores?). Es posible que por haber mayores pueblos mitimaes en el valle Azapa sujetos al mandón Cariapassa, este ha debido fijar su residencia en el valle mencionado, pero que de alguna manera el valle de Tacna también estaba dentro de sus funciones.

\section{CONCLUSIONES}

Insistir en una organización andina prehispánica dual, además de que este concepto está más relacionado con la época Inka, ausente en el valle de Tacna, sobre la base de las informaciones de encomiendas y ante la falta de evidencias, no parece acertado. Hidalgo (2004) describe que el valle de Tacna hasta 1538-1540 estuvo gobernado por dos caciques principales en una estructura unitaria dual. Si antes de la primera visita del valle, el Marqués Francisco Pizarro prometió una cédula con un número mayor de indios a Pedro Pizarro y poco después redujo el número porque “...conviene que vuestro repartimiento sea moderado para dar en él indios a otra persona por estar el dicho vuestro repartimiento en comarca de poder mejor servir al pueblo de villa hermosa..." (Barriga, 1939, p. 41); entonces de allí se desprende que la otra "mitad" de pueblos del valle de Tacna, o si se quiere la cantidad restante con sus principales debieran tener un mandón que responda por ellos; esta división, antes que una organización dual andina, fue una solución ajustada por la necesidad y conveniencia del español, por lo menos para la zona de Tacna.

¿Cuál hubiese sido el resultado de los estudios actuales sobre la organización local, si el Marqués Pizarro concedía toda la encomienda de Tacna originalmente prometida a su primo Pedro? Estamos seguros que allí habría constado como único mandón a Istaca y 1400 indios con sus principales; por tanto, una sola comunidad indígena con un solo representante. Por el momento, salvo nuevas investigaciones, la dualidad prehispánica o las parcialidades primitivas "hanasaya" y "urinsaya" señaladas para el valle de Tacna no refleja una antigua organización andina; y la división en dos encomiendas en el mismo valle no hace sino tomar las informaciones históricas tempranas con mucha cautela.

Finalmente, se piensa que en el reparto de esta encomienda ha primado la riqueza y extensión de las tierras agrícolas, a pesar de la deficiente cantidad de agua para estas labores. Obviamente, en un área donde no hay desembarcaderos óptimos para naves de gran calado, minas de oro o rebaños con carneros de 
la tierra, el encomendero va a preferir tierras de cultivo y Pedro Pizarro, en ese aspecto, se vio beneficiado por ser pariente del "marqués", al otorgarle el valle medio hacia la cabecera de Tacna con la cuenca hidrográfica a disposición.

\section{REFERENCIAS}

Arellano, C. (1989). Hanan/urin: Reflexiones acerca de un viejo concepto dual inka y su aplicación en el Chinchaysuyu. 50 Años de Estudios Americanistas en la Universidad de Bonn, 30, 473-493.

Barriga, V. (1939). Documentos para Historia de Arequipa, 1534-1558. Tomo I. Arequipa: Editorial La Colmena S. A.

Barriga, V. (1940) .Documentos para la Historia de Arequipa, 1534-1575. Tomo II. Arequipa: Editorial La Colmena S. A.

Barriga, V. (1955). Documentos para la Historia de Arequipa, 1535-1580. Tomo III. Arequipa: Editorial La Colmena S. A.

Cavagnaro, L. (1986). Materiales para la Historia de Tacna. Cultura Autóctona. Cooperativa San Pedro de Tacna, Fondo de Desarrollo Cultural.

Cavagnaro, L. (1994). Materiales para la Historia de Tacna. Dominación hispana (S. XVII). Cooperativa San Pedro de Tacna, Fondo de Desarrollo Cultural.

Cúneo, R. (1977). Historia de la fundación de la ciudad de San Marcos de Arica. Obras Completas Tomo 5, Vol. IX.

Chacaltana, S. (2015). Regional Interfaces between Inca and Local Communities in the Colesuyo Region of Southern Peru. (tesis de postgrado en Antropología). College of the University of Illinois at Chicago.

Gordillo, J. (1989). Estudio Arqueológico en Sitajara, Yabroco y Susapaya (cabeceras del valle de Sama) (tesis de pregrado).
Universidad Católica Santa María de Arequipa.

Gordillo, J. (2014). Ensayo sobre el rol de los yungas y altiplánicos en los valles occidentales de Tacna. La vida y la Historia, III (2): 30-37.

Gordillo, J. y Bolaños, A. (2019). La producción arquitectónica del sitio Inca de Moqi, Tacna-Perú. Arkinka 286, 74-87.

Hidalgo, J. (1996). Relaciones protohistóricas interétnicas entre las poblaciones locales y altiplánicas en Arica. La integración sur andina: cinco siglos después, (161173).

Hidalgo, J. (2004). Historia andina en Chile. Editorial Universitaria, Chile.

Hidalgo, J. y Focacci, G. (1986). Multietnicidad en Arica, S. XVI. Evidencias etnohistóricas y arqueológicas. Chungará 16-17,137-147.

Julien, C. (1989). Guano and resource control in sixteenth-century Arequipa. Andean Ecology and Civilization, (185-231).

Rostworowski, M. (1977). La estratificación social y el Hatun Curaca en el mundo andino. Histórica I (2):249-285.

Trimborn, H. (1975). Investigaciones arqueológicas en los valles del Caplina y Sama (Dep. Tacna, Perú). Navarra España: Editorial Verbo Divino.

Vaughan, S. (2013). Architectural Analysis of the Moqi Inca Site. Tesis de pregrado. The Archaeological Studies Program Department of Sociology and Archaeology. University of Wisconsin.

Vela, C. (2004). La cerámica Cerro Los Hornos, presencia Inka en la costa de Tacna, sur del Perú. Chungará, Volumen Especial (935-942). 